\title{
The Child's Craniopharyngiomas About 11 Cases and Review of the Literature
}

\author{
T. Mesbahi, A. Rafiq, L. Panu, O. Jamal, A. Naja and A. Lakhdar
}

\section{ABSTRACT}

Craniopharyngioma is a benign and rare intracranial tumor. In children, the clinic is characterized by the semiological, neurological, ophthalmological, and endocrine tripod. Medical imaging, represented by computed tomography (CT) and especially magnetic resonance imaging (MRI), has revolutionized the approach to the diagnosis of the latter. It has a slow evolution and likely to recur late. Thus, prolonged clinicalradiological follow-up is important to affirm a cure. The aim of this study is to highlight the epidemiological, clinical, paraclinical, therapeutic and evolutionary characteristics of craniopharyngiomas in children. This work consisted of a retrospective study of 11 pediatric cases of this tumor collected in the Neurosurgery Department of the IBN ROCHD University Hospital in Casablanca between January 2011 and January 2017. In this series, the child's craniopharyngiomas accounted for a rate of $45.8 \%$ of all these tumors. The average age of our patients was 9.09 years, with a sex ratio of 0.37 . The average time to diagnose was 10 months. The picture was dominated by $100 \%$ visual disturbances, signs of intracranial hypertension at $63.63 \%$ and endocrine disorders at $36.36 \%$. The lesion was intra and suprasellar seat in $91 \%$ of cases and suprasellar seat in $9 \%$. Tumor size was less than $4 \mathrm{~cm}$ in $\mathbf{5 4 . 5 3 \%}$ of cases. Hydrocephalus is observed in $27.27 \%$ of cases. The fronto-pterrional modus operandi was the most used $72 \%$ of cases. Total exeresis was achieved in $9.1 \%$ of cases. Overall trends were favorable in $72 \%$ of cases without noting mortality. Tumor progressive recovery was observed in $9.1 \%$ of cases, but there were no recurrences. Given its location, the treatment of craniopharyngioma can lead to significant consequences in children affecting the long-term quality of life, hence the importance of post-operative follow-up and multidisciplinary management.

Keywords: Benign tumor, child, craniopharyngioma, multidisciplinary management, neurosurgery.
Submitted: September 5, 2021

Published: October 14, 2021

ISSN: $2593-8339$

DOI: $10.24018 /$ ejmed.2021.3.5.1045

\section{T. Mesbahi}

Centre Hospitalier Universitaire IBN ROCHD Casablanca, Morocco.

A. Rafiq*

Centre Hospitalier Universitaire IBN ROCHD Casablanca, Morocco.

(e-mail:faresmeryemrafiq@gmail.com) L. Panu

Centre Hospitalier Universitaire IBN ROCHD Casablanca, Morocco.

O. Jamal

Centre Hospitalier Universitaire IBN ROCHD Casablanca, Morocco.

A. Naja

Centre Hospitalier Universitaire IBN ROCHD Casablanca, Morocco.

A. Lakhdar

Centre Hospitalier Universitaire IBN ROCHD Casablanca, Morocco.

*Corresponding Author

\section{INTRODUCTION}

Craniopharyngioma is a benign and rare tumor of the central nervous system, slow-moving, developing in the saddler or suprasellar region. It has between 8 and 13\% of brain tumors in children the clinical picture leading to diagnosis often associated endocrine disorders with signs of intracranial hypertension [2]. Different therapeutic alternatives are described: surgery, radiotherapy and more rarely intra cavity chemotherapy, without a clear consensus. The after-effects after initial management are almost systematic. They are essentially endocrine for almost all patients [4], visual, neurological, and neurocognitive.

The aim of this work is to highlight the epidemiological, clinical, clinico-pathological, para-clinical, therapeutic, and evolutionary characteristics of craniopharyngioma in children by comparing the results with those of the literature.

\section{PATIENTS AND METHODS}

This is a work that focused on a series of 11 cases of craniopharyngiomas in children under the age of 16 , collected in the Neurosurgery Department of the IBN ROCHD University Hospital in Casablanca over a 6-year period from January 2011 to January 2017.

\section{RESULTS}

Of the 24 cases of craniopharyngiomas reported in the Neurosurgery Department of the IBN ROCHD Hospital over the 6-years period from January 2011 to January 2017, 11 cases were children under the age of 16 .

The average hospitalization was 1.83 cases per year. The average age of our patients was 9.09 years. The sex ratio is 0.37 .

The diagnosis time is an average of 10 months. Most patients were polysymptomatic, manifestations dominated by visual disorders in $100 \%$ of cases, intracranial hypertension syndrome $63.63 \%$ and a minority of endocrine disorders $36.36 \%$

Clinical signs of antehypophysis insufficiency were found 
in 04 patients whose failure to thrive was the master endocrine symptom. We noted in 01 patient's clinical signs of hypothyroidism, 01 patient had tasteless diabetes.

Almost present visual signs are often the first clinical manifestations of the tumor. The ophthalmological examination performed in all our patients revealed visual impairment, including 05 severe cases.

Brain radiological exploration of CT scan and magnetic resonance imaging (MRI) without and with injection of contrast products (PDCs) was performed in all our patients. These two examinations revealed 10 cases of intra and suprasellar tumors: $91 \%$ and 01 case of suprasellar tumor or $9 \%$. The different tumor dimensions were noted identically for both examinations, ranging from $2 \mathrm{~cm}$ to more than $6 \mathrm{~cm}$ of large axis.

TABLE I: BREAKDOWN OF DIFFERENT TUMOR COMPONENTS

\begin{tabular}{cc}
\hline \hline Tumor Structure & Number of Cases \\
\hline \hline Solido-cystic component & 09 \\
Purely fleshy component & 00 \\
Purely cystic component & 02 \\
Calcifications & 03 \\
\hline \hline
\end{tabular}

Note that MRI is a crucial means in the pre-operative check-up to obtain a detailed description and analysis of the tumor, on the other hand CT is important in cases of calcified tumors.

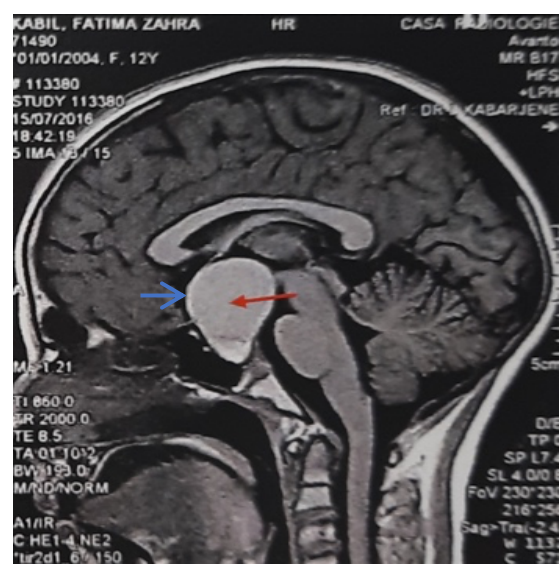

Fig. 1. MRI in weighted T1 sequence with gadolinium injection in sagittal cut showing a craniopharyngioma double-component basal fleshy (red arrow), strongly enhanced by the PDC in a homogeneous manner, and cystic in hypersignal whose wall is enhanced in crown Blue arrow), intra and suprasellar seat with extension Higher to the V3 filling the optochiasmatic tank and compressing the optical chiasma.

Endocrine explorations have not been carried out in all our pre-operative patients due to the urgency of some cases. Only 07 of the 11 cases were explored, of which 03 were cases with normal balance sheets and 04 cases had a dissociated hormonal deficit balance. 03 cases with ACTH deficiency, 02 cases with GH deficiency, 02 cases with Thyrotropic deficit and 01 cases of hyperprolactinemia.

All our patients benefited from a direct response to the tumor, including 03 patients who had previously benefited from ventriculo-peritoneal derivation for hydrocephalus. The first approach was unilateral fronto-pterrional in 08 cases, under unilateral frontal in 02 cases and pterrional in 01 cases.

The sometimes-difficult location of this tumor forces the Neurosurgeon to settle for subtotal or partial exeresis, despite the aim of surgery in these cases being as complete an exeresis as possible of the craniopharyngioma.

Medical treatment is hormone replacement mainly in cases of antehypophysis deficit and insipid diabetes.

Only 02 patients in our series received conventional radiotherapy after initially partial exeresis. No cases of chemotherapy as an adjuvant treatment of the tumor have been reported.

Post-operative hospitalization in the intensive care unit is imperative for close and close monitoring of the operation by the teams of Neurosurgeons and resuscitators. For our patients, there were 08 cases of simple immediate postoperative, 01 cases with neurological worsening made of generalized seizures and 03 cases presented an insipid Diabetes.

A brain control CT scan was performed in all our patients.

There were no cases of immediate mortality

The median decline in our series was 2 years. Of our 11 patients, only 10 were screened (01 lost). We did not identify any cases of long-term mortality

All our patients developed a regression of intracranial hypertension syndrome. Seizures were controlled on anticonvulsant therapy in our patient. Visual acuity was improved in 04 patients, and we noted the persistence of blindness in 05 patients. Evolution was stationary in 02 patients.

Only 01 patient was surgically resumed in post-operative, it is an evolutionary tumor recovery with a delay of 3 years (initially operated in another structure).

We did not identify any cases of tumor recurrence.

\section{DISCUSSION}

Since craniopharyngioma is on the diencephalic stage, it can develop at any level of the infundibulo-pituitary axis, from the sphenoidal body to the cinereum tuber. It disrupts the functioning of several surrounding structures by its compressive and invasive expansions, causing sometimes fatal damage caused by its surgical exeresis. Its contiguity with several noble structures including the hypothalamicpituitary axis, the hypothalamus at the top, above the floor of the 3rd ventricle, the monro holes and backwards the Aqueduct of Sylvius. At the top and back its relationship with the mamillary tubers whose lesion causes memory problems. This tumor can also extend backwards from the sellable dorsum to the cerebral peduncles, where it meets the branches of the basilar trunk, the posterior cerebral arteries, and the emergence of the common oculomotor nerve (III). The craniopharyngioma can reach the bridge and bulb by filling the pre-pontic and pre-bulb tanks in its giant forms. Downstairs, its relationship with the sphenoidal body and the turcic saddle that it can expand or destroy. The craniopharyngioma responds laterally to the cavernous sinuses where the internal carotid artery and the external oculomotor nerve (VI) move, not sheltering the pathetic (IV) that travels to the outer wall of this sinus. This tumor can repress, compress, or encompass the oculomotor nerves, its lateral expansion can sometimes reach the central gray nuclei or temporal lobes.

Histologically, two subtypes of craniopharyngiomas, the Adamantinian subtype and the papillary subtype according to 
the WHO classification, are described. Transitional and mixed forms are also described.

Adamantinian craniopharyngioma, common in children, derives from embryological residues in the Rathke pouch. This type contains different solid and cystic components. Calcifications are common, a more invasive type locally and often adhering to adjacent tissues [12].

Some authors found a better 5-year survival rate for papillary craniopharyngiomas by comparing them to adamantinians and mixed ones; and a much higher perioperative mortality rate in Adamantinians tumors. Because craniopharyngioma is histologically benign, rare cases of malignant transformation and remote tumor spread of the primary tumor have been reported. The mechanism of dissemination is done either by seeding along the first surgical pathway, or by migrating tumor cells via arachnoid spaces or Virchow-Robin spaces.

Craniopharyngioma is the most common intracranial tumor in children whose origin is non-glial. It accounts for $10 \%$ of all intracranial tumors, $18 \%$ of sub tentorial tumors and $55 \%$ of suprasellar tumors [1].

Exceptional before the age of 2 years, craniopharyngiomas are regularly distributed with 3 frequency peaks: between 7 and 13 years, 20 and 25 years, 60 and 65 years.

In our series, the average age at diagnosis was 9.09 years, ranging from 2 to 16 years with a peak in disease frequency between 8 and 12 years. The other series of literature give a predominance of the disease in the end of the first decade with a peak at 6-8 years and another at 14-16 years. Much of the literature gives a slight male predominance about 55\% according to [10].

In our series, we found a female predominance, sex ratio to 0.37 or 08 female patients and 03 male.

In our series, the average diagnosis time was 7 months, with extremes ranging from 3 months to 4 months. In the literature, the average is 20 months and appears shorter in children than in adults. This period usually takes one week to several years.

The relative proportions of tell-tale signs vary widely depending on the series in the literature. The tell-tale signs are more often neurological in children, then ophthalmological, while endocrine signs come in third because patients are often overlooked or unrecognized. At the time of diagnosis, the clinical picture in our series is like that described in the literature.

Intracranial hypertension is the preferred mode of revelation of the disease in children and is present in more than half of cases.

The location of the tumor in the saddle region, its relationship to visual pathways and its impressive evolutionary potential has an impact on vision. For this reason, ophthalmological examination is an integral part of the preoperative assessment of craniopharyngiomas. This examination assesses the severity of the visual impairment, gives a rough idea of the size and location of the tumor in relation to the optic pathways. Especially for further followup, have a reference to visual function in relation to the surgical act. In the literature, the frequency of visual impairments is very high and estimated at $60 \%$ in children.

In our series, the tumor was revealed by blindness in 05 children or $45.45 \%$ of cases.
Endocrine disorders are the third most clinical sign of craniopharyngioma. In the literature, they account for $40 \%$ of cases under interrogation. The endocrine disorders described in the literature are stunting (the main endocrine sign in children), pubertal delay (infrequent sign) and insipid diabetes resulting in polyuro-polydipsic syndrome in less than $20 \%$ of cases according to the literature [10].

In our series, we have $36.36 \%$ of cases of failure to thrive, $9.1 \%$ of tasteless diabetes and $9.1 \%$ of hypothyroidism.

The current excellent way to study tumor and peri-tumor anatomy is Magnetic Resonance Imaging (MRI). It exceeds the scanner in the fine study of tumor reports with the optic pathways, pituitary stem and sellar diaphragm. The study of the relationship of the tumor with the cerebral parenchyma is of paramount importance [10]. Some of the anatomical structures surrounding the tumor may be damaged (rolled, pent-up, stretched, stretched, or engulfed) by the tumor. MRI can also be used to explore, like CT scans, the relationships of the tumor with elements of the Willis polygon.

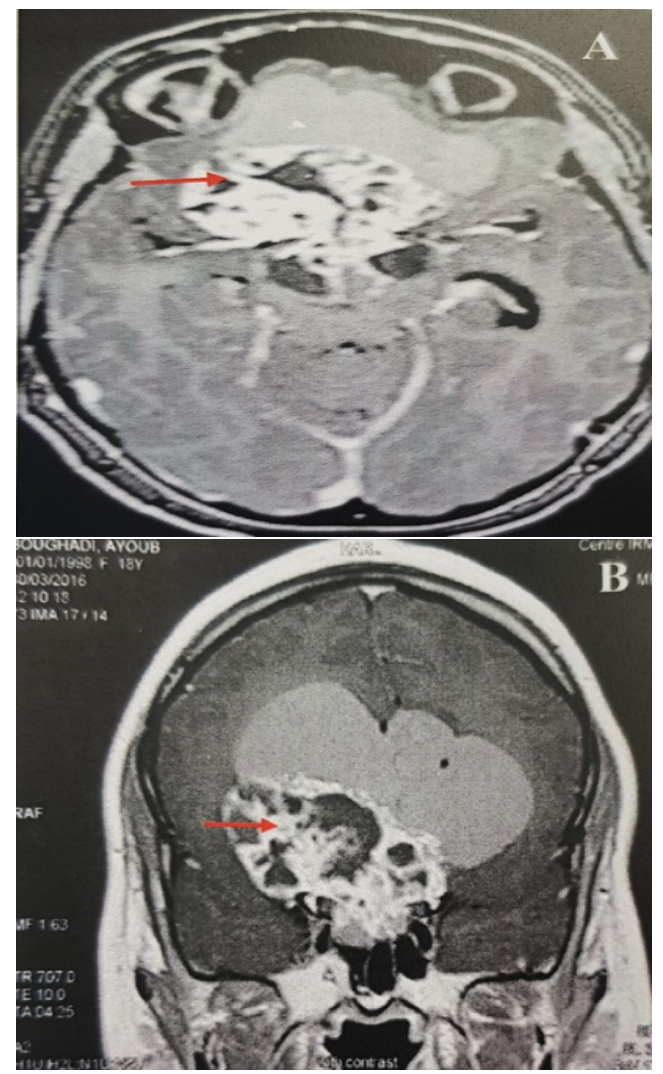

Fig. 2. Preoperative MRI showing in an axial cut (A) injected T1 sequence and coronal cut (B) showing an extended craniopharyngioma in the frontal sub (red arrow), third ventricle and posterior cerebrally pit.

A complete preoperative endocrine check-up is essential, but due to the diagnostic delay, some patients have a neurological or ophthalmological picture requiring an urgent surgical procedure, therefore, endocrine explorations have not always been performed in preoperative. Endocrine deficiency can complicate all types of craniopharyngiomas regardless of their location, because by its embryological origin, the tumor is always related to the hypothalamicpituitary tract.

Craniopharyngiomas are an essential surgical and endocrine therapeutic problem. A strategy must be determined to limit the risk of mortality, morbidity, and 
recurrence at the same time. Despite histologically benign lesion, but with high evolutionary potential, this tumor is curable provided the exeresis is total. The ideal for the treatment of this extra cerebral benign tumor should be a complete extirpation. Often impossible due to the invasion of an essential anatomical structure.

The approaches first described for craniopharyngioma exeresis are used isolation or in combination, or even successively. We will cite the main direct first approaches used in craniopharyngioma surgery which are:

- The fronto-pterrional approach (used in $72.72 \%$ of the cases in our series).

- The sub frontal approach (used in 2 cases in our series, or $18.18 \%$ of our patients), is the most used in the literature and historically it is the first way to have allowed satisfactory exeresis.

- The pterrional approach (used in a single case of our series, or $9.1 \%$ of our patients).

- The sub temporal approach.

- The Trans ventricular approach; and

- The Trans-sphenoidal approach.

In our series, none of our patients were approached by any of the last three pathways.

The invasiveness of the craniopharyngioma is the determining factor on which the quality of the exeresis depends. It is generally not possible to remove an invasive tumor from the nerve structure in which it enters. For complete exeresis of the tumor, the invaded parenchyma is sacrificed because there is no good cleavage plan between the tumor and the healthy parenchyma. The possibility of practicing a complete exeresis is very variously appreciated in the literature, no doubt the results depend on the surgeon and his technical tray.

In our series, the exeresis was appreciated by a brain control scanner. It was total in 01 cases $(9.1 \%)$, subtotal in 07 cases $(63.63 \%) 03$ cases $(27.27 \%)$.

Bypass remains an emergency means to control possible intracranial hypertension in craniopharyngiomas associated with hydrocephalus while waiting for exeresis surgery.

Stereotactic techniques, such as the installation of a tank catheter or simple evacuating punctures, allow the evacuation of tumor cysts associated with or not the intracavity injection of chemicals or radioactive products.

In our series, a patient benefited from the Rickam Reservoir set-up. Punctures were found to be difficult due to the thick nature of the cystic fluid.

Interventional endoscopy is a low-risk and minimally invasive therapeutic modality that allows for perfect interventional control.

Neuronavigation has brought perfect precision and safety in craniopharyngioma surgery, in addition to the use of microsurgical and neuro-endoscopic techniques.

The role of radiation therapy is still under discussion, especially in children, whose developing brain is very sensitive to the harmful effect of ionizing radiation. This treatment exposes to the risk of radio necrosis, especially visual pathways, post-radial arteritis, radio-induced tumors, and especially intellectual deficit in children. It is recommended for children over the age of 4 .

We have three radiotherapy techniques that are: conventional, endocavitary and stereotactic.

In our series, 01 patients $(9.1 \%)$ received conventional radiotherapy after a partial initial exeresis).

The initial management of craniopharyngiomas depends on clinical presentation. Signs of intracranial hypertension and progressive visual impairment require urgent neurosurgical treatment of hydrocephalus and/or decompression of the cystic portion or tumor. The resolution of intracranial hypertension leads to stabilization or even improvement in visual impairment in $2 / 3$ of patients.

Two first pathways are possible to operate craniopharyngiomas: a high, transcranial pathway, and a low, trans-sphenoidal path. The choice of pathway first depends on the location, appearance and size of the tumor and the surgeon's experience and preferences.

Early and delayed, post-therapeutic surveillance serves a threefold purpose [10], [11]: to control the possible disappearance of the tumor, to monitor the resumption of the expansive process and to assess the harmful side effects of treatments. This surveillance must be clinical, ophthalmological, biological but above all radiological (CT and MRI), as many recurrences of craniopharyngiomas remain silent for a long time [11]. MRI is the exam of choice for post-operative surveillance. It allows to evaluate the extension of the exeresis, to differentiate from the second month the scar fibrosis that does not take the contrast, from a tumor recurrence that takes it. Even in the absence of clinical call signs, at a distance from the procedure, a radiological examination (CT and MRI with injection) will be performed systematically from the second month, and every year for the five years following the procedure [10],[11] and then every two years until 10 years.

In our series, we did not list any post-operative deaths. Visual acuity improved in 04 patients, remained stationary in 02 cases, and evolution was marked by persistence of blindness in 05 cases.

In our series, tasteless diabetes is revealed in immediate post-operative in $27.27 \%$. Regardless of the first route, tasteless diabetes remains the main post-operative endocrine complication. It most often appears within hours or days of surgery. Much more rarely, it settles several months after treatment. It can be transient or permanent.

Disorders of higher functions can occur after surgical treatment, they affect memory, concentration and especially intelligence.

Sequellar motor deficits exist in 2 to $20 \%$ of cases [10]; sensory disorders especially anosmia; sequellar epilepsy is noted in 5 to $20 \%$ of cases (controlled with anticonvulsants); and the disorder of consciousness may be seen as a risk to which any major intracranial surgery is exposed.

Craniopharyngioma is a slow-growing tumor susceptible to late recurrence, requiring prolonged follow-up to affirm its cure.

This should include regular consultations, ophthalmological and endocrine checks, and imaging examinations with a fine study of the intra and suprasellar region. This was not the case for many of our patients for several reasons, particularly socio-economic difficulties, and remoteness.

The median decline in our series was 2 years. Of our 11 patients, only 10 were screened and 01 lost sight. 
Recidivism is the major risk in the evolution of craniopharyngiomas [12]. They are slow-moving, evolutionary recovery can be late, so postoperative monitoring should be very prolonged: an MRI per year is recommended for the first five years, then one every two years for at least 10 years. Ophthalmological monitoring is essential, as many recurrences manifest themselves as visual impairment. It is an evolution of the fragments, sometimes microscopic left in place by the surgery and not a "real" recurrence. The risk of recurrence depends on the quality of the exeresis in radical surgery. In the case of incomplete exeresis, radiotherapy reduces this risk to a level close to that observed after a total exeresis, at the cost of its own complications [12]. Most recurrences occur in the first five years [13].

After subtotal exeresis without adjuvant radiotherapy, most tumors recur or resume growth in less than one year [14].

In our series, only 10 out of 11 patients were reviewed over the long term with a median decline of 2 years.

\section{CONCLUSION}

Craniopharyngiomas are benign and rare, epithelial tumors derived from residues from the Rathke pouch, which occur most often in children or adolescents. The call signs are visual, neurological, and endocrine. The advent of minimally invasive therapeutic modalities and innovative radiotherapy techniques has brought significant advances in the treatment of craniopharyngioma. Not to mention neuronavigation, all these techniques constitute the essential arsenal of the Neurosurgeon and have significantly reduced per-operative mortality. Given its location in the sellar region, the treatment of craniopharyngioma can lead to significant sequelae (visual, neuropsychological, and endocrine) affecting the quality of life in the long term, hence the need for postoperative follow-up coupled with multidisciplinary management by the Neurosurgeon and other specialists depending on the importance of the after-effects.

\section{REFERENCES}

[1] R. van Effenterre and A. Boch, "Craniopharyngioma," Ann Endocrinol 68:412-21, 2007

[2] H. L. Muller, "Childhood craniopharyngioma," Pituitary 16:56-67, 2013.

[3] J. Flitsch, J. Aberle and T. Bhurkhardt, "Surgery for paediatric craniopharyngiomas: is less more?" J Pedietr Endocrinol Metab 28:2733, 2015.

[4] E. M. Erfurth, "Endocrine aspects and sequel in patients with craniopharyngioma," J Pediatr Endocrinol Metab 2015; 28:16-26.

[5] A. Poretti, M. A. Grotzer, K. Ribi, E. Schönle and E. Boltshauser, "Outcome of craniopharygioma in children: long-term complications and quality of life," Dev Med Child Neurol 2004; 46:220-9.

[6] J. Duff, F. B. Meyer, D. M. Ilstrup, E. R. Laws Jr, C. D. Schleck and B. W. Scheithauer, "Long-term outcomes for surgically resected craniopharyngiomas," Neurosurgery 46:291-302, 2000.

[7] N. Karavitaki, S. Cudlip, C. B. Adams and J. A. Wass, "Craniopharyngiomas," Endocr Rev 27:371-97, 2006.

[8] G. R. Bunin, T. S. Surawicz, P. A. Witman, S. Preston-Martin, F. Davis and J. M. Bruner, "The descriptive epidemiology of craniopharyngioma," J Neurosug 89:547-51, 1998.

[9] W. Q. Huang, S. J. Zheng, Q. S. Tian, J. Q. Huang, Y. X. Li, Q. Z. Xu and W. C. Zhang, "Statistical analysis of central nervous system tumors in China," Journal of Neurosurgery 56(4), 555-564, 1982.
[10] M. Choux, G. Lena and L. Genitori, "Craniopharyngioma of the child," Neurosurgery 37, Suppl:1-174, 1991.

[11] R. Van Effenterre and A. L. Boch "Craniopharyngiomas of adult and child: study of a surgical series of 106 consecutive cases," Neurosurgery 43940:187-211, 1997.

[12] V. C. Prabhu and H. G. Brown, "The pathogenigis of craniopharyngiomas," Childs Nerv Sys 21:622-7, 2005.

[13] A. Pierre-Kahn, R. Brauner, D. Denier, C. Saint Rose, M. Gangemi, R. Rappot and J. F. Hirch, "Treatment of the child's craniopharyngiomas retrospective analysis of 50 observations," Arch Fr Pediatr 45: 163167, 1998.

[14] D. C. Stripp, A. Maity, A. J. Janss, J. B. Belasco, Z. A. Tochner, J. W. Goldwein and H. K. G. Shu, "Surgery with or without radiation therapy in the management of craniopharyngiomas in children and young adults," Int J. Radiot Oncol Biol Phys 58(3):714-720, 2004.

[15] V. Stagno, J. Mugamba and P. Senyonga, "Presentation, pathology, and treatment outcome of brain tumors in 172 consecutve children at Cure children's hospital of Uganda. The predominance of the visible diagnosis and the uncertainties of epidemiology in Sub-Saharan Africa," Childs Nerv Syst 30(1):137-146, 2014. 